\title{
Intraocular Pressure Changes during Femtosecond Laser-Assisted Cataract Surgery: A Comparison between Two Different Patient Interfaces
}

\author{
Chiara De Giacinto $(\mathbb{D}$, Rossella D'Aloisio $(\mathbb{D}$, Alessandro Bova $(\mathbb{D}$, Tommaso Candian, \\ Alberto Armando Perrotta, and Daniele Tognetto \\ Eye Clinic, Department of Medicine, Surgery and Health Sciences, University of Trieste, Trieste 34129, Italy \\ Correspondence should be addressed to Rossella D’Aloisio; ross.daloisio@gmail.com
}

Received 6 June 2019; Revised 23 August 2019; Accepted 5 September 2019; Published 25 September 2019

Academic Editor: Antonio Queiros

Copyright (c) 2019 Chiara De Giacinto et al. This is an open access article distributed under the Creative Commons Attribution License, which permits unrestricted use, distribution, and reproduction in any medium, provided the original work is properly cited.

Purpose. The aim of this retrospective cohort study was to evaluate intraocular pressure (IOP) changes during femtosecond laserassisted cataract surgery (FLACS) using two different patient interface systems. Methods. 116 eyes of 116 patients scheduled for cataract surgery were divided into 2 groups: group 1 (61 eyes) and group 2 (55 eyes) underwent FLACS using Catalys Laser with fluid interface (liquid optics interface, LOI) and LenSx Laser with curved interface and soft contact lens (SoftFit), respectively. IOP was assessed using a portable rebound tonometer $\left(\right.$ Icare $\left.^{\circledR}\right)$ preoperatively, after docking, immediately after surgery, at one and seven days postoperatively. Results. In group 1, the mean IOP $( \pm \mathrm{SD})$ was $14.1 \pm 0.4 \mathrm{mmHg}$ before surgery, $33.2 \pm 1.1 \mathrm{mmHg}$ after docking, and $21.4 \pm 0.9 \mathrm{mmHg}$ immediately after surgery. In group 2, the mean IOP was $13.8 \pm 0.4 \mathrm{mmHg}$ before surgery, $24.2 \pm 1.4 \mathrm{mmHg}$ after docking, and $20.2 \pm 1.2 \mathrm{mmHg}$ immediately after surgery. After the docking procedure, a statistically significant increase in IOP from the baseline was found in both groups $(p<0.001)$. Moreover, no statistically significant difference in IOP measured at 1 and 7 days postoperatively was observed compared with the preoperative values $(p>0.05)$ using both laser platforms. No intraoperative and postoperative complications were observed. Conclusions. FLACS suction phase resulted in a transient increase of IOP in both groups, especially with the LOI system, and it is probably related to the greater pressure of a suction ring and suction generated through the vacuum, independently from the effect of femtosecond laser itself.

\section{Introduction}

Femtosecond laser technology, firstly introduced as a new technique for creating lamellar flaps in laser-assisted in situ keratomileusis (LASIK) in 2001, has been rapidly developed for cataract surgery, showing several advantages in different surgical steps, such as corneal incisions, anterior capsulotomy, and lens fragmentation $[1,2]$.

All FLACS systems rely on interfaces for the docking phase, which is one of the most delicate steps, allowing good suction and the final success of laser treatment [1]. Docking has three functions: the optical coupling allowing efficient delivery of the laser beam into the ocular tissues, the ocular mechanical stabilization during laser application, and the accurate acquisition of bi- and tri-dimensional images ensuring the correct position of the corneal incisions $[3,4]$.
However, an increase in IOP during the docking step has been previously reported because of corneal compression during the applanation process [5-7]. The first published studies that compared two different femtosecond lasers have evaluated IOP changes in vivo and ex vivo animal models, describing a higher increase in IOP using the flat applanation interface in comparison with the curved one [5-7].

Fluid-filled interfaces have been developed to cope with applanation interface-related rise in IOP and have shown to cause less corneal folds and a lower IOP increase than curved contact lens interface [8].

The liquid optics interface (LOI) of Catalys ${ }^{\circledR}$ Laser, similarly to ultrasonic examination devices, uses water immersion to minimize the impedance mismatch between the transducer and the eye [9]. LOI permits less pressure on 
the cornea as there is no direct force or deformation of the corneal structure [8].

On the other hand, LenSx ${ }^{\circledR}$ Laser has a one-piece patient interface connected to the counter-balanced laser objective head and is characterized by a curved applanation interface combined with the most recent upgrade of soft lens-assisted interface (SoftFit ${ }^{\mathrm{TM}}$ ) [10].

The aim of this study was to investigate IOP changes during and after FLACS in human eyes which underwent surgery performed by the same surgeon, comparing two completely different patient interfaces: Catalys ${ }^{\circledR}$ Liquid Optics Interface and LenSx ${ }^{\circledR}$ SoftFit $^{\mathrm{TM}}$ interface.

\section{Materials and Methods}

This retrospective cohort study included 116 eyes of 116 patients who underwent FLACS and intraocular lens (IOL) implantation performed by the same experienced surgeon (D. T.) at the Department of Medicine, Surgery and Health Sciences, University of Trieste, Italy, between December 2015 and October 2017. This retrospective observational study adhered to the tenets of the Declaration of Helsinki, and our Institutional Review Board approved the retrospective consecutive chart review.

The inclusion criteria were the following: medium lens opacities according to the Lens Opacities Classification System III (LOCS III) [11], good pupil dilation $(\geq 5.0 \mathrm{~mm})$, and age above 18 years.

The exclusion criteria were the following: glaucoma, corneal opacities, previous surgical treatment within 6 months, and ocular inflammatory conditions.

Informed consent was obtained from all eligible subjects for the use of their data.

A total of 61 eyes were treated with Catalys ${ }^{\circledR}$ Precision Laser System (Johnson \& Johnson, Santa Ana, CA, USA) with LOI (group 1) and 55 eyes with LenSx ${ }^{\circledR}$ Laser System (Alcon, Fort Worth, TX, USA) with curved contact lens SoftFit ${ }^{\mathrm{TM}}$ interface (group 2).

2.1. Patient Interface Docking Procedure. In group 1, the Catalys ${ }^{\circledR}$ Precision Laser system with the LOI module used a two-piece process: a suction ring that has contact only with the sclera (patient interface dimensions: an outer diameter of $22.3 \mathrm{~mm}$ and an inner diameter of $13.5 \mathrm{~mm}$ ) and a disposable lens linking the suction ring to the surgical system. Firstly, the suction ring was located perfectly central on the sclera of the eye. After some minimal adjustments, the suction ring vacuum was created leading to the connection between the suction ring and patient eye. Secondly, a balanced salt solution (BSS) was used to fill the suction ring volume. Finally, the suction ring was adjusted and connected to the laser head docking. Regarding group 2, the LenSx ${ }^{\circledR}$ system used a single-piece patient interface (patient interface dimensions: an outer diameter of $19.8 \mathrm{~mm}$ and an inner diameter of $12.5 \mathrm{~mm}$ ) that was attached to the machine and then docked to the eye using the joystick and the video image on the graphic user interface. After patient docking, both LenS $\mathrm{x}^{\circledR}$ and Catalys ${ }^{\circledR}$ platforms performed OCT imaging of the eye which is $2 \mathrm{D}$ and $3 \mathrm{D}$, respectively. The images were analysed for treatment planning and for the final steps of the femtosecond laser treatment, such as anterior capsulorhexis, nuclear fragmentation, and corneal incisions.

2.2. Intraocular Pressure Reading Protocol. The day before surgery, all patients had a complete ophthalmic evaluation including best corrected visual acuity examination, slit lamp biomicroscopy, IOP measurements assessed with a portable rebound tonometer (Icare ${ }^{\circledR}$, TA01I, Icare Finland Oy), biometry, central corneal thickness, and anterior chamber evaluation.

IOP was measured with Icare ${ }^{\circledR}$ just before the docking phase ( 5 minutes before), after the docking procedure (just after that the eye was undocked, about 1 minute later), and just after the end of surgery, at 1 day and 7 days postoperatively, during follow-up visits. Each time, the average of 5 IOP measurements was considered and registered on our electronic medical records. All patients were placed in the sitting position in the operating bed for the pressure reading procedures. Before surgery, all eyes were anesthetized with oxybuprocaine hydrochloride $0.4 \%$. In addition, phenylephrine and tropicamide $10 \%+0.5 \%$, tropicamide $1 \%$, and anti-inflammatory eye drops of diclofenac sodium $0.1 \%$ were used before surgery as well. After the patient was docked to the system, the OCT imaged the anterior chamber, and the system created a $3 \mathrm{D}$ treatment plan with the Catalys ${ }^{\circledR}$ system and $2 \mathrm{D}$ with the LenSx ${ }^{\circledR}$ system. After laser application and removal of the suction, manual surgical cataract procedure and IOL implantation in the capsular bag were performed in the same operating room.

2.3. Statistical Analysis. Continuous data were expressed as the mean \pm standard deviation (SD). Statistical analysis was performed using IBM ${ }^{\circledR}$ SPSS Statistics v 20.0 software (SPSS Inc., Chicago, Illinois, USA). Repeated measures ANOVA with linear trend analysis were performed to evaluate the effect of surgical phases on IOP values. Contrast analysis was performed to evaluate differences of each parameter from the previous measurement. A $p$ value less than 0.05 was considered statistically significant.

\section{Results and Discussion}

3.1. Results. A total of 116 eyes of 116 patients were considered.

The demographic and clinical characteristics at the baseline are reported in Table 1.

No statistically significant difference was found between the two groups in terms of clinical cataract grading, preoperative IOP levels, anterior chamber depth, central corneal thickness, and axial length (Table 1).

Group 1 was composed of 61 eyes with a mean age of $72.4 \pm 7.6$ years (ranged between 47 and 89 ), and in group 2, 55 patients with a mean age of $71.5 \pm 13.9$ years were evaluated (ranged between 23 and 89). In group 1, the mean IOP $( \pm$ SD) was $14.1 \pm 0.4 \mathrm{mmHg}$ before surgery, $33.2 \pm 1.1 \mathrm{mmHg}$ after 
TABLE 1: Demographic and clinical characteristics at the baseline.

\begin{tabular}{lccccc}
\hline \multicolumn{5}{c}{ Patient demographics and preoperative clinical status } \\
Parameter & \multicolumn{4}{c}{ Group 1 } & \multicolumn{2}{c}{ Group 2 } & R value $(<0.01)$ \\
\hline Age $(\mathrm{y})$ & Mean \pm SD & Range & Mean \pm SD & Range & 0.69 \\
Male : female (n) & $72.4 \pm 7.6$ & 47,89 & $71.5 \pm 13.9$ & 23,89 & - \\
Left eye : right eye (n) & $33: 28$ & - & $27: 28$ & - & - \\
ACD $(\mathrm{mm})$ & $37: 24$ & - & $32: 23$ & - & 0.42 \\
CCT $(\mu \mathrm{m})$ & $3.06 \pm 0.24$ & $2.61,3.6$ & $3.17 \pm 0.38$ & $2.58,4.06$ & 0.38 \\
AL $(\mathrm{mm})$ & $560.9 \pm 35.6$ & 525,630 & $555.2 \pm 28.5$ & 509,634 & 0.76 \\
Preop IOP $(\mathrm{mmHg})$ & $23.84 \pm 1.49$ & $21.56,28.9$ & $23.92 \pm 1.36$ & $21.93,27.3$ & 0.15 \\
\hline
\end{tabular}

ACD: anterior chamber depth; CCT: central cornea thickness; AL: axial length.

docking, and $21.4 \pm 0.9 \mathrm{mmHg}$ just after surgery. In group 2, the mean IOP was $13.8 \pm 0.4 \mathrm{mmHg}$ before surgery, $24.2 \pm 1.4 \mathrm{mmHg}$ after docking, and $20.2 \pm 1.2 \mathrm{mmHg}$ after surgery (Table 2, Figure 1).

In both groups, a statistically significant increase in IOP after the suction procedure was found from the baseline $(p<0.001$; Table 2, Figure 1). Moreover, in both groups, the IOP measured at 1 and 7 days postoperatively did not show any statistically significant difference with the preoperative values ( $p>0.05$; Table 2$)$.

A statistically significant difference between the two groups in terms of rise in IOP was found only after the docking phase with a higher value in group 1 (Table 2).

In group 1, the mean total suction time was 2 minutes and 50 seconds \pm 54 seconds; the laser capsulotomy was completed in 1.5 seconds using the following treatment parameters: pulse energy range $4-5 \mu \mathrm{J}, 4.9 \mathrm{~mm}$ diameter capsulotomy, $5 \mu \mathrm{m}$ horizontal spot spacing, and $10 \mu \mathrm{m}$ vertical spot spacing; the capsulotomy needed little energy, only $0.7 \mathrm{~J}$ in each eye. The mean lens laser fragmentation time was 48 seconds \pm 27 seconds. The lens was segmented into quadrants or quadrants softened patterns with the following treatment parameters: grid spacing range $350 \mu \mathrm{m}-800 \mu \mathrm{m}$, segmentation repetition range $5-10,10 \mu \mathrm{m}$ horizontal spot spacing and $40 \mu \mathrm{m}$ vertical spot spacing, anterior pulse energy range $8-10 \mu \mathrm{J}$, and $10 \mu \mathrm{J}$ posterior pulse energy. The mean total energy lens fragmentation was $12.8 \pm 4.9 \mathrm{~J}$.

In group 2, the mean total suction time was 2 minutes and 45 seconds \pm 34 seconds; the laser capsulotomy was completed in 2.5 seconds using the following treatment parameters: pulse energy range $7-8 \mu \mathrm{J}, 4.9 \mathrm{~mm}$ diameter capsulotomy, and $5 \mu \mathrm{m}$ tangential and layer spot separation; the capsulotomy needed only $0.6 \mathrm{~J}$ of energy in each eye. The mean lens laser fragmentation time was 46 seconds \pm 20 seconds without any statistically significant difference if compared to group $1(p=0.67)$. The lens was segmented into cylinders or matrix grid patterns with 12 to $13 \mu \mathrm{J}$ posterior/anterior pulse energy.

Laser-assisted cataract treatments were successfully performed in all cases. No intraoperative and postoperative complications were observed, including suction loss during the laser treatment or posterior capsule rupture, and no adverse events occurred. Furthermore, no patient reported amaurosis.
IOL implantation in the capsular bag was performed in all cases.

3.2. Discussion. As previously described, a rise in IOP during the docking phase in FLACS was related to the use of a suction ring and to the corneal applanation during cone device coupling. Indeed, advances in interface technology have been developed, and new patient interfaces with and without the corneal applanation procedure have been introduced $[1,12]$.

Catalys Laser has a liquid optics interface that does not need applanation of the cornea. The liquid patient interface has been associated with a lower IOP increase than the corneal applanation systems. Moreover, real-time IOP measurements assessed in porcine eyes underwent surgery with the Catalys platform were lower if recorded in the vitreous cavity instead of the aqueous humour [13].

Contact lens SoftFit ${ }^{\mathrm{TM}}$ interface of the LenSx platform has been recently introduced, as well. The soft contact lens, made up of a hydrogel material, closely matches the curvature of the cornea with minimal distortions, preserving the natural corneal curvature and fixing the eyeball. This type of interface gives higher control and stability reducing eye movements if compared to LOI, thus decreasing also the pressure to be employed on the eye by the surgeon [10].

At the beginning of the FLACS procedures, holding and manually placing the suction ring on the eye, sometimes without a lid speculum, can be very challenging in patients who have small palpebral fissures or difficult fixation. This manoeuvre is easier with LenSx due to the smaller diameter of the limbal suction ring [10].

In our work, the surgeon had a great experience with both lasers. A similar ease of the docking phase was found in both groups, likely due to high confidence of the experienced surgeon with different laser systems.

Similarly to our findings, the current SoftFit interface has been associated with an increase of IOP by approximately $16 \mathrm{mmHg}$, without short- or long-term complications directly related to the higher levels of IOP from the baseline [10].

Our results demonstrated a statistically significant transient increase in IOP from the baseline values in both patient interface systems, just after the docking phase and suction ring removal. Immediately after cataract surgery, 
TABLE 2: Mean IOP data at the different study time points of the two groups.

\begin{tabular}{|c|c|c|c|c|c|c|c|}
\hline \multicolumn{8}{|c|}{ Mean IOP data at the different study time points } \\
\hline \multirow[b]{2}{*}{ Study time points } & \multicolumn{3}{|c|}{ Group 1} & \multicolumn{4}{|c|}{ Group 2} \\
\hline & Mean $\pm \mathrm{SD}(\mathrm{mmHg})$ & $p$ value $^{1}(<0.01)$ & $p$ value $^{2}(<0.01)$ & Mean \pm SD $(\mathrm{mmHg})$ & $\begin{array}{l}p \text { value }^{1} \\
(<0.01)\end{array}$ & $\begin{array}{c}p \text { value }^{2} \\
(<0.01)\end{array}$ & $p$ value $^{3}(<0.01)$ \\
\hline Before suction & $14.1 \pm 0.4$ & 1.0 & - & $13.8 \pm 0.4$ & 1.0 & - & 0.68 \\
\hline After vacuum & $33.2 \pm 1.1$ & $<0.001$ & - & $24.2 \pm 1.4$ & $<0.001$ & - & $<0.001$ \\
\hline After phaco & $21.4 \pm 0.9$ & $<0.001$ & $<0.001$ & $20.2 \pm 1.2$ & $<0.001$ & 0.67 & 0.395 \\
\hline 1 day after & $14.0 \pm 0.4$ & 1.0 & $<0.001$ & $12.7 \pm 0.3$ & 1.0 & $<0.001$ & 0.01 \\
\hline 7 days after & $13.9 \pm 0.3$ & 1.0 & $<0.001$ & $13.2 \pm 0.3$ & 1.0 & $<0.001$ & 0.09 \\
\hline
\end{tabular}

Before suction: IOP measured before laser treatment; after vacuum: IOP measured just after vacuum turned off and suction ring removed on lying patients; after phaco: IOP measured after cataract surgery; 1 day after: IOP measured at 1 day after surgery; 7 days after: IOP measured at 7 days after surgery. $p$ value ${ }^{1}$ : statistical significance of IOP values compared to preoperative values; $p$ value ${ }^{2}$ : statistical significance of IOP values compared to values obtained after suction phase; $p$ value ${ }^{3}$ : statistical significance of the IOP difference between the two groups at each study time points. Group 1: liquid optics interface. Group 2: SoftFit ${ }^{\mathrm{TM}}$ Patient Interface.

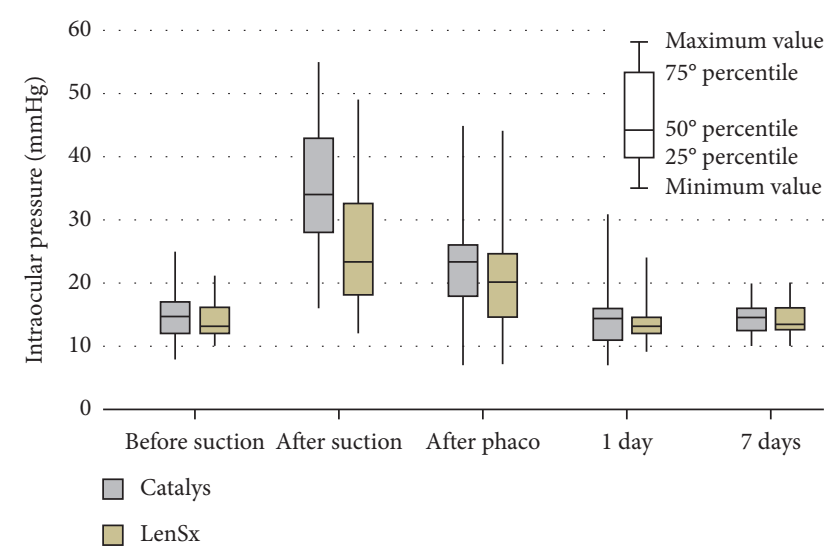

Figure 1: Intraocular pressure values measured at different time points during FLACS performed with two different devices: Catalys and LenSx. The box is determined by the central mean as well as the $25^{\text {th }}$ and $75^{\text {th }}$ percentiles. The whiskers represent the minimum value $\left(0^{\text {th }}\right.$ percentile $)$ and the maximum value $\left(100^{\text {th }}\right.$ percentile).

IOP values decreased and came back to preoperative values at 1 day postoperatively.

Moreover, the rise in IOP during the suction phase was significantly higher in the LOI system versus the contact lens interface.

The highest levels of IOP induced by the vacuum using the soft lens interface was approximately $11 \mathrm{mmHg}$ above the predocking IOP/the preoperative value, while in the LOI system, the vacuum-induced IOP rose by about $20 \mathrm{mmHg}$ compared to baseline/compared to presurgery. Previous studies described very high IOP levels using flat and curved contact lenses for corneal flap creation with a femtosecond laser. Vetter et al. in their experimental study with enucleated porcine globes found much higher mean IOP levels than those reported in our work [7]. Talamo et al. [14] measured intraocular pressure ex vivo in porcine and human cadaver eyes and found a mean IOP rise of $32.4 \mathrm{mmHg} \pm 3.4$ in the curved contact lens interface group and $17.7 \mathrm{mmHg} \pm 2.1 \mathrm{mmHg}$ in the LOI group during the suction phase.

Schulz et al. [15], in a prospective clinical trial, found a mean IOP level of $27.7 \mathrm{mmHg} \pm 5.5$, after removing the LOI interface, which is comparable to those obtained in our study in terms of amount of IOP increase in the liquid optics system.

On the contrary, we observed a lower rise in IOP with the SoftFit ${ }^{\mathrm{TM}}$ if compared with data reported in the literature regarding curved interfaces $[7,14]$. It is also true that they considered rigid curved interfaces with a rigid contact lens, conversely to our work. The soft contact lens interface sets with more stability of the eye with a decreased risk for suction loss, differently from the rigid one that does not perfectly match the natural ocular shape, thus resulting in IOP rise $[10,14]$.

LOI has a mechanical contact only outside the limbus, on the overlying conjunctiva, where anatomic alterations are minimal, thus minimizing globe deformation and corneal irregularities. Anyway, LOI has been associated with pressure rises during the docking process because of the force downward of the suction ring by the additional weight of the disposable lens. In addition, micromovements of the eye could further increase the IOP during suction $[13,16]$.

We did not expect additional IOP increase from the BBS used because of the openings of the suction ring that can be a way of leak when the solution is displaced by the lens during docking.

Another interesting element to consider is the possibility of cavitation bubbles to cause an additional increase of IOP with their action of expansion of the lens volume and capsular bag [17].

Less energy to fragment a softer cataract could be correlated to a lower production of bubbles and a lower intraocular pressure. However, our sample included homogeneous grade of cataract, and we did not consider this aspect.

Our work has the unique angle of describing two completely different designs of interfaces that have shown to be associated with a minimal and transient increase in IOP, without any anatomical changes and any iatrogenic damage to the optic disc, confirming the safety of FLACS with excellent final anatomical and functional outcomes and no IOP-related postoperative complications.

Sudden increases of IOP, even though not very high, can be dangerous for the ocular structure [8]. Anyway, in our 
study, although a rise of IOP during suction was registered immediately after the suction procedure, IOP levels decreased immediately after the docking phase and suction ring removal and IOP values at 1 and 7 days after the surgical procedure were not statistically significant different than preoperative values.

In a nonrandomized prospective case series, the authors reported a higher transient increase of IOP after vacuum undocking in glaucomatous subjects than in those without the disease, but it seemed to be well tolerated in the short period in both groups [8].

\section{Conclusions}

To the best of our knowledge, no other study has compared the IOP variations in vivo during FLACS comparing these two types of interface systems: the fluid-filled interface versus the current soft contact lens system.

The main limitations of our study are its retrospective nature and the lack of IOP readings during the laser treatment to better understand its effect on IOP changes. Ibarz et al. [13] measured IOP in porcine eyes, every five seconds during the femtosecond procedure using a direct cannulation system to the anterior chamber. However, the real-time IOP in porcine eyes did not show any effect of the laser treatment on IOP modifications, and we believe that the transient increase in IOP during the docking phase is probably related to the greater pressure of the LOI suction ring and of suction generated through the vacuum, independently from the action of the femtosecond laser itself.

\section{Data Availability}

All data will be available if requested to the corresponding author.

\section{Disclosure}

This work was presented in part at the $21^{\text {st }}$ Winter Meeting of the European Society of Cataract and Refractive Surgeons, Maastricht, Netherlands, February 2017. Chiara De Giacinto and Rossella D'Aloisio are co-first authors.

\section{Conflicts of Interest}

The authors declare that there are no conflicts of interest regarding the publication of this article.

\section{Authors' Contributions}

Chiara De Giacinto and Rossella D'Aloisio contributed equally to this work.

\section{References}

[1] S. Trikha, A. M. J. Turnbull, R. J. Morris, D. F. Anderson, and P. Hossain, "The journey to femtosecond laser-assisted cataract surgery: new beginnings or a false dawn?," Eye, vol. 27, no. 4, pp. 461-473, 2013.
[2] N. J. Friedman, D. V. Palanker, G. Schuele et al., "Femtosecond laser capsulotomy," Journal of Cataract \& Refractive Surgery, vol. 37, no. 7, pp. 1189-1198, 2011.

[3] Z. Nagy, A. Takacs, T. Filkorn, and M. Sarayba, "Initial clinical evaluation of an intraocular femtosecond laser in cataract surgery," Journal of Refractive Surgery, vol. 25, no. 12, pp. 1053-1060, 2009.

[4] F. H. Hengerer, M. Mittelbronn, M.-L. Hansmann, G. U. Auffarth, and I. Conrad-Hengerer, "Femtosecond laserassisted capsulotomy: histological comparison of four different laser platforms," Journal of Refractive Surgery, vol. 33, no. 10, pp. 670-675, 2017.

[5] S. S. Chaurasia, F. L. Gimeno, K. Tan et al., "In vivo real-time intraocular pressure variations during LASIK flap creation," Investigative Opthalmology \& Visual Science, vol. 51, no. 9, pp. 4641-4645, 2010.

[6] J. L. Hernandez-Verdejo, M. A. Teus, J. M. Roman, and G. Bolivar, "Porcine model to compare real-time intraocular pressure during LASIK with a mechanical microkeratome and femtosecond laser," Investigative Opthalmology \& Visual Science, vol. 48, no. 1, pp. 68-72, 2007.

[7] J. M. Vetter, M. P. Holzer, C. Teping et al., "Intraocular pressure during corneal flap preparation: comparison among four femtosecond lasers in porcine eyes," Journal of Refractive Surgery, vol. 27, no. 6, pp. 427-433, 2011.

[8] E. Darian-Smith, A. R. Howie, R. G. Abell et al., "Intraocular pressure during femtosecond laser pretreatment: comparison of glaucomatous eyes and nonglaucomatous eyes," Journal of Cataract \& Refractive Surgery, vol. 41, no. 2, pp. 272-277, 2015.

[9] C. J. Pavlin, J. A. McWhae, H. D. McGowan, and F. S. Foster, "Ultrasound biomicroscopy of anterior segment tumors," Ophthalmology, vol. 99, no. 8, pp. 1220-1228, 1992.

[10] T. Roberts, M. Lawless, G. Sutton, and C. Hodge, "Update and clinical utility of the LenSx femtosecond laser in cataract surgery," Clinical Ophthalmology, vol. 10, pp. 2021-2029, 2016.

[11] L. T. Chylack Jr, J. K. Wolfe, D. M. Singer et al., "The lens opacities classification system III," Archives of Ophthalmology, vol. 111, no. 6, pp. 831-836, 1993.

[12] H. S. Uy, K. Edwards, and N. Curtis, "Femtosecond phacoemulsification: the business and the medicine," Current Opinion in Ophthalmology, vol. 23, no. 1, pp. 33-39, 2012.

[13] M. Ibarz, J. L. Hernández-Verdejo, G. Bolívar, P Tañá, J. L Rodríguez-Prats, and M. A Teus, "Porcine model to evaluate real-time intraocular pressure during femtosecond laser cataract surgery," Current Eye Research, vol. 41, no. 4, pp. 507-512, 2016.

[14] J. H. Talamo, P. Gooding, D. Angeley et al., "Optical patient interface in femtosecond laser-assisted cataract surgery: contact corneal applanation versus liquid immersion," Journal of Cataract \& Refractive Surgery, vol. 39, no. 4, pp. 501-510, 2013.

[15] T. Schultz, I. Conrad-Hengerer, F. H. Hengerer, and H. B. Dick, "Intraocular pressure variation during femtosecond laser-assisted cataract surgery using a fluid-filled interface," Journal of Cataract \& Refractive Surgery, vol. 39, no. 1, pp. 22-27, 2013.

[16] R. Yeoh, "Practical differences between 3 femtosecond phaco laser platforms," Journal of Cataract \& Refractive Surgery, vol. 40 , no. 3, p. 510, 2014.

[17] C. Khng, M. Packer, I. H. Fine, R. S. Hoffman, and F. B. Moreira, "Intraocular pressure during phacoemulsification," Journal of Cataract \& Refractive Surgery, vol. 32, no. 2, pp. 301-308, 2006. 


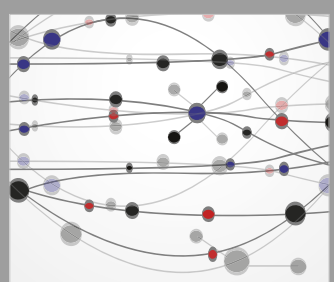

The Scientific World Journal
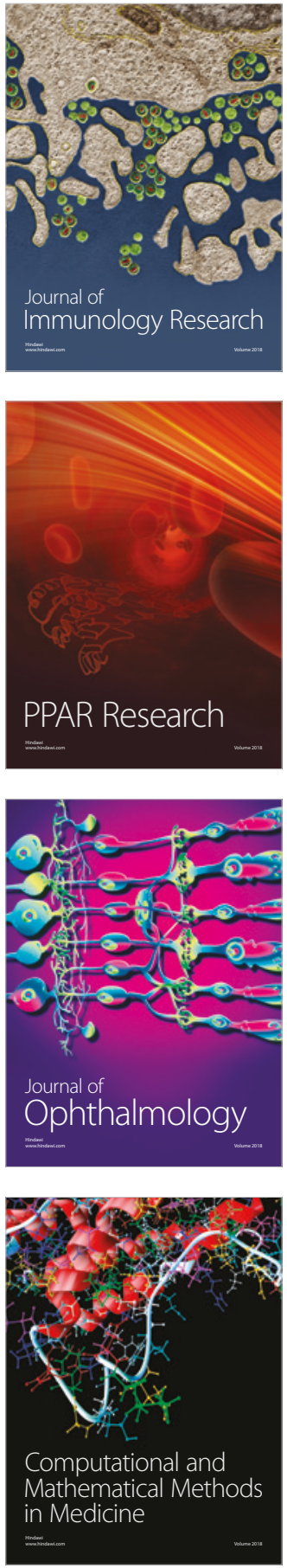

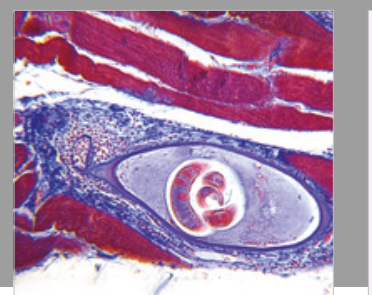

Gastroenterology Research and Practice

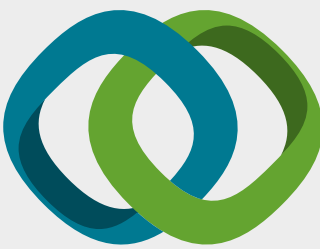

\section{Hindawi}

Submit your manuscripts at

www.hindawi.com
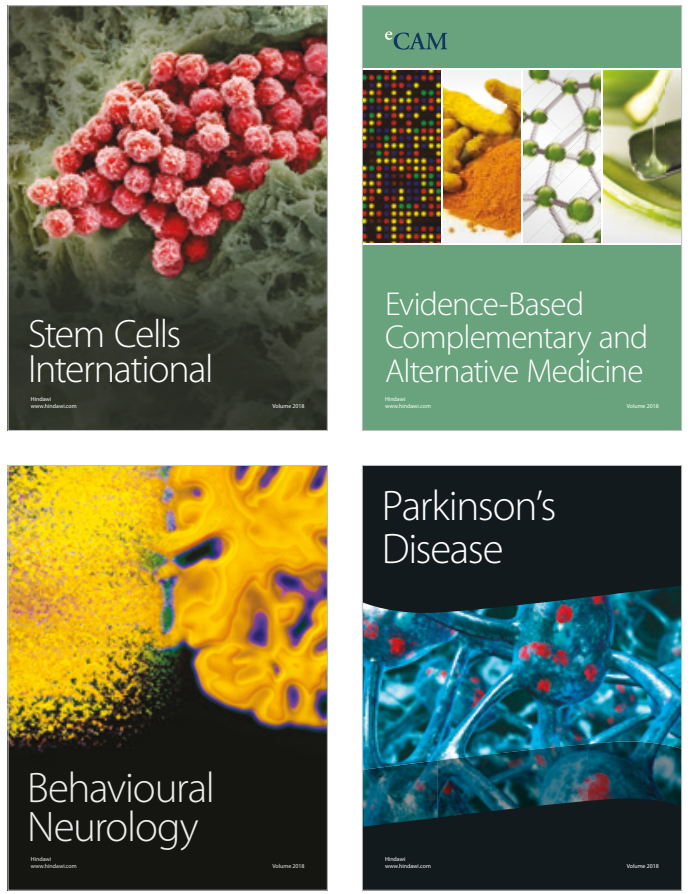

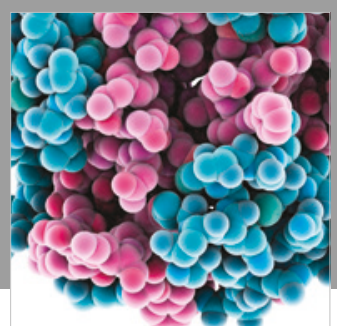

ournal of

Diabetes Research

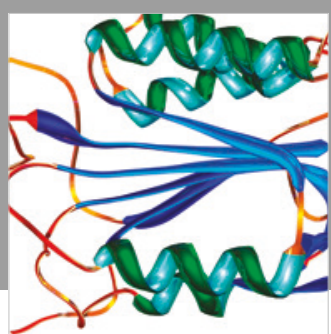

Disease Markers
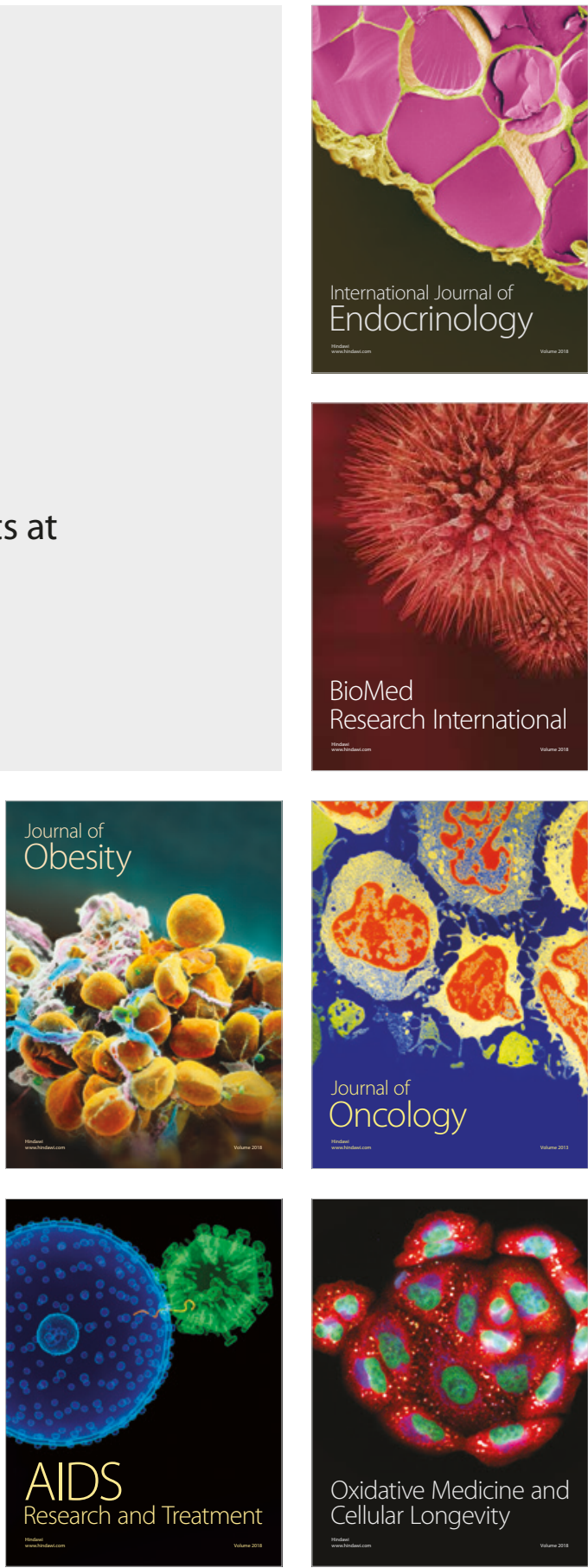